\title{
UNE REMARQUE SUR LA FORMULE DE RESIDUS
}

\author{
PAR WEISHU SHIH
}

Communicated by Joseph J. Kohn, December 2, 1969

La théorie de Leray sur les résidus a fait l'objet d'études par divers auteurs, notamment: Norguet, Dolbeault, Sorani. Certaines conditions de restriction sont imposées sur la sous-variété en considération dans leurs études. Récemment Griffiths a établi la formule des résidus dans le cas algébraique où la sous-variété $W$ de codimension $q$ arbitraire soumise aux restrictions suivantes:

(i) $W$ est nonsingulière.

(ii) $W$ est homologue par la dualité de Poincaré à la qième classe de Chern d'un fibré ample sur la variété ambiante $V$ algebraique non singulière.

Le but de ce travail est d'établir ce même résultat de Griffiths, mais en supprimant ces deux restrictions. Plus précisément, on a le

THÉORÈme. Soit $V$ une variêté Kählêrienne compacte, $W$ un sousespace analytique, compacte, de codimension complexe $q \geqq 1$. Alors il existe $\psi$, une forme $L^{1}, d u$ type $(q, q-1)$ sur $V$ vérifiant les conditions suivantes:

(1) La restriction $\left.\psi\right|_{V-W}$ de $\psi$ sur le complémentaire $V-W$ est $\mathfrak{C}^{\infty}$ et vérifie $d^{\prime} \psi=0$.

(2) La restriction $\left.d^{\prime \prime} \psi\right|_{V-W}$ se prolonge d une forme $\mathfrak{C}^{\infty}$ sur $V$ qui est homologue par la dualité de Poincaré a-W.

(3) Au voisinage de $W$ on a

$$
\psi \sim O(2 q-1)
$$

c'est-d-dire dans un voisinage tubulaire suffisemment petit de $W$, on a

$$
\left|\gamma^{2 q-1} \cdot \psi\right|<\infty
$$

où $\gamma(x)$ est le germe de fonction près de $W$ défini par la distance de $x$ d $W$ (par rapport à la métrique Riemannienne associée a la structure Kählérienne de V).

(4) Pour toute chaine différentiable $Z$ de $V$ de dimension réelle $p$ dont l'intersection avec $W$ est transversale, et toute forme $\mathbb{e}^{\infty}$ fermée $\omega$ sur $V$ de degrê $p-2 q$, on a la formule de résidus:

AMS Subject Classifications. Primary 5380, 5760.

Key Words and Phrases. Several complex variables, a generalisation of Griffiths' residue operators. 


$$
\lim _{\epsilon \rightarrow 0} \int_{\partial \tau_{e}(W) \cap Z} \psi \wedge \omega=\int_{W \cap Z} \omega
$$

où $\tau_{\epsilon}(W)$ désigne un voisinage tubulaire de $W$ de rayon plus petit que $\epsilon>0$, et $\partial \tau_{\epsilon}(W)$ son bord.

REMARQUE. La forme $\psi$ est déterminée explicitement par la structure Kählérienne de $V$. Cette unicité nous permet d'appeler $\psi$ comme "la forme du résidu associé à $W$ " de la variété Kählérienne $V$. Dans le cas où $V$ est une variété analytique complexe compacte quelconque, la forme $\psi$ existe encore et est vérifiée; à l'exception du fait qu'elle est de type $(q, q-1)$ et $d^{\prime} \psi=0$, les autres conditions du théorème, en particulier la formule de résidus.

Il m'eût été impossible de faire cette étude, si je n'avais pas reçu les aides importantes et constantes du Professeur Deligne. Je tiens à lui exprimer ma plus profonde gratitude. Je remercie aussi le Professeur Unterberger pour ses nombreux conseils et discussions.

\section{REFERENCES}

1. P. Dolbeault, Theory of residues and homology, Séminaire P. Lelong Analyse 1968/69, Lecture Notes in Math., Springer-Verlag, Berlin and New York (to appear).

2. P. Griffiths, (a) Lecture notes $1967 / 68$, Princeton University, Princeton, N. J., (b) Some results on algebraic cycles on algebraic manifolds, Algebraic Geometry, Oxford Univ. Press, Oxford, 1969, pp. 93-191.

3. J. Leray, Le calcul differential et intégral sur une variêté analytique complexe (probleme de Cauchy. III), Bull. Soc. Math. France 87 (1959), 81-180. MR 23 \#A3281.

4. F. Norguet, Sur la théorie des rêsidus, C. R. Acad. Sci. Paris 248 (1959), 20572059. MR $21 \# 5195$.

5. G. Sorani, Sui résidui delle forme differenziali di una varietà analitica complessa, Rend. Mat. e Appl. (5) 22 (1963), 1-23. MR 27 \#6262.

Institut des Hautes Études Scientifiques, Bures-Sur-Yvette, France 